\title{
High Performance Fourier Transform Ion Cyclotron Resonance Mass Spectrometry Via a Single Trap Electrode
}

\author{
Victor H. Vartanian and David A. Laude \\ Department of Chemistry and Biochemistry, University of Texas at Austin, Austin Texas, USA
}

\begin{abstract}
An open-ended cylindrical cell with a single annular trap electrode located at the center of the excitation and detection region is demonstrated for Fourier transform ion cyclotron resonance mass spectrometry. A trapping well is created by applying a static potential to the trap electrode of polarity opposite the charge of the ion to be trapped, after which conventional dipolar excitation and detection are performed. The annular trap electrode is axially narrow to allow the creation of a potential well without excessively shielding excitation and detection. Trapping is limited to the region of homogeneous excitation at the cell centerline without the use of capacitive coupling. Perfluorotributylamine excitation profiles demonstrate negligible axial ejection throughout the entire excitation voltage range even at an effective centerline potential of only $-0.009 \mathrm{~V}$. High mass resolving power in the single-trap electrode cell is demonstrated by achievement of mass resolving power of $1.45 \times 10^{6}$ for benzene during an experiment in which ions created in a high pressure source cubic cell are transferred to the low pressure analyzer single-trap electrode cell for detection. Such high performance is attributed to the negligible radius dependent radial electric field for ions cooled to the center of the potential well and accelerated to less than $60 \%$ of the cell radius. An important distinction of the single-trap electrode geometry from all previous open and closed cell arrangements is exhibition of combined gated and accumulated trapping. Because there is no potential barrier, all ions penetrate into the trapping region regardless of their translational energy as in gated trapping, but additional ions may accumulate over time, as in accumulated trapping. Ions of low translational kinetic energy are demonstrated to be preferentially trapped in the single-trap electrode cell. In a further demonstration of the minimal radial electric field of the single-trap electrode cell, positive voltages can be applied to the annular trap electrode as well as the source cell trap electrode to achieve highly efficient transfer of ions between cells. (J Am Soc Mass Spectrom 1995, 6, 812-821)
\end{abstract}

$\mathrm{T}$ The development of Fourier transform ion cyclotron resonance (FTICR) mass spectrometry $[1,2]$ has resulted in a numerous array of trapped-ion cells with various electrode arrangements of cubic [3], orthorhombic [4, 5], cylindrical [6-9], hyperbolic [10-13], or multiple-electrode design [1325]. Each of the designs has been adapted to FTICR because of an inherent advantage or improvement related to the quality of the quadrupolar potential, magnitude of the radial trapping field, or homogeneity of the excitation field. Hyperbolic electrode cells were adapted to FTICR to achieve the highest mass resolving power possible due to the approximate independence of measured cyclotron frequency on ion position achieved in a nearly pure quadrupole potential. Cubic cells were elongated to improve ion capacity, reduce space-charge, increase sensitivity, and reduce the radial electric field $[5,26]$. Grounded screens were inter-

Address reprint request to David A. Laude, Department of Chemistry and Biochemistry, The University of Texas at Austin, TX 78712. posed between the trap electrodes and the cell interior to reduce the radial electric field in the cell interior by up to 2 orders of magnitude [21]. Segmented electrode designs with resistive networks were developed to allow fine tuning of the voltages applied to the electrodes to linearize the trapping or excitation electric fields to approximate electrodes of infinite extent [16-18] or to minimize higher order fields that couple the axial and radial modes of ion motion $[19,20]$ that lead to the production of harmonic and combination frequencies [27]. Although these advances in cell design have resulted in improved FTICR performance, typically they have come at the expense of increased number of cell electrodes or added electrical circuitry. Consequently, some cells may not be as robust as simpler six electrode cells used in the conventional FTICR experiment.

Previous improvements in the traditional closed cell do not address the basic deficiencies of the electrode geometry, the difficulty of introduction of a charged particle beam efficiently through narrow conductance 
limits without contaminating or charging the trap electrodes, and the necessity of passing the ion beam in close proximity to paramagnetic materials [28]. Openendcap trapped-ion cell configurations originally were used in the physics community to improve particle beam injection efficiencies in studies of electron and atomic beams, plasma interactions, and antimatter confinement [29-33], although analysis of the trapping frequency rather than the cyclotron frequency was often performed.

The flexibility of the collinear electrode geometry allows any number and combination of electrodes to be used in the construction of new trapped-ion cells. Gabrielse et al. [34] used compensation electrodes adjacent to the trap electrodes and collinear with the excitation and detection electrodes to eliminate anharmonicity in the trapping well without affecting the well depth and thereby obtained greater trapping frequency precision for antiproton mass measurements. Such a cell allows trapping well anharmonicity to be electrically "tuned out" or compensated without affecting the well depth by adjustment of the relative voltages on the trap and compensation electrodes. Recent publications indicate that preparations are underway to produce antihydrogen by simultaneous trapping and interacting antiprotons and positrons in two adjacent trapping wells formed in the same cell [35].

The introduction of open geometry trapped-ion cells for FTICR by Beu and Laude [36] permitted conventional mass analysis to be performed with trap electrodes parallel to excitation and detection electrodes in an entirely collinear electrode geometry. The open cell configuration is now being examined for improved quadrupolar potential [37] and is increasingly adopted by various FTICR researchers to increase external ion injection efficiency, improve gas conductance in the cell, eliminate the formation and charging of dielectric surfaces, and eliminate ion trajectory perturbations from ions that pass in proximity to paramagnetic electrode surfaces [38-41]. FTICR performance also improves as a consequence of increased excitation field homogeneity because the excitation field does not terminate as abruptly on trap electrodes positioned orthogonally to the excitation electrodes.

Beu and Laude also made use of the collinear geometry to capacitively couple the excitation electrodes to the trap electrodes for improved excitation field uniformity and reduced axial ejection [42]. Recently the open cell has been adapted for simultaneous trapping of positive and negative ions [43]. In addition, compensation (or guard) electrodes have been used in conjunction with the trap electrodes to reduce the radial electric field in the cell [44] analogous to the screened cell of Wang and Marshall [21].

To date all closed or open geometry trapped-ion cells form a potential well by using two trap electrodes. In this article, a cell is demonstrated with a single annular trap electrode located at the center of the excitation and detection region that creates a trap- ping well by applying a static potential of a polarity that is opposite the charge of the ion to be trapped. There exists historical precedence to this approach of trapping ions. Kingdon relied on a negative voltage applied to a small wire or filament to trap positive ions to reduce space-charge and improve performance in early vacuum tubes. Later, endcaps were added to prevent ions from escaping axially, and this became known as the Kingdon trap [45]. Similarly the orbitron vacuum pump trapped electrons around a positively biased filament $[46,47]$. Wire ion guides also rely on trapping ions in a radial potential well formed by applying voltage to the wire opposite the ion charge, which results in increasing radial focusing, mass range extension, and sensitivity in time-of-flight experiments of ions of low kinetic energy or large kinetic energy distributions [48-50]. Electron beam ion traps create radial potential depressions formed by an intense electron beam in which highly charged positive ions can accumulate after formation by repeated ionization [51] and the electron beam can be used to manipulate ions in an FTICR cell [52]. Finally, nested traps of open cylindrical geometry have been used for positron and antiproton confinement $[33,40]$ in which a single trap electrode biased oppositely to one trapped charge is nested within two other conventionally biased trap electrodes of the same charge as the other ion.

The single-trap electrode cell to be described affords superior FTICR performance by using a combination of applied electric fields to virtually eliminate the axial ejection of ions and by generating a reduced radial electric field throughout a significant portion of the trapping volume. The cell also yielded the highest FTICR mass resolving power among all the trapped-ion cells we have evaluated. An additional feature of the cell is the exhibition of combined gated [53-59] and accumulated $[56,60,61]$ trapping. Because no electrostatic barrier exists, ions can be externally generated and injected into the cell without discrimination on the basis of translational energy as in gated trapping. However, continuous ion injection into the cell can occur simultaneously as in accumulated trapping. Such an electrode configuration is possible only with the collinear open cell geometry, which illustrates that appropriate electrode selection and location can be used to improve cell performance without increasing cell complexity.

\section{Experimental}

The cylindrical single-trap electrode cell excitation and detection electrodes were constructed of 0.030 -in. wall thickness OFHC (oxygen-free high-conductivity) copper tubing sectioned and mounted at a 52-mm-i.d. aluminum frame. The overall cell dimensions were 75-mm length, $50-\mathrm{mm}$ diameter, with a $3.3-\mathrm{mm}$ aluminum ring of $46.5-\mathrm{mm}$ i.d. and $48.0-\mathrm{mm}$ o.d. suspended as the trap electrode in the center of the excitation and detection region. A 1-mm air gap sepa- 
rated the outer surface of the trap electrode from the inner surface of the excitation and detection electrodes.

The cell was appended to the low pressure analyzer side of a commercial Waters Extrel (Madison, WI) dual cell flange assembly within an FTMS-2000 spectrometer (Extrel) that included a 3-T, 150-mm warm bore superconducting magnet controlled by a Nicolet (Madison, WI) 1280 data station that executes FTMS version 6.0 .1 by using a conventional $50-\mathrm{mm}$ edge length closed cubic trapped-ion cell mounted on the source side of the flange. A pressure differential in excess of 2 orders of magnitude was achieved across the 2-mm conductance limit, which also serves as a trap electrode for the cubic cell. In addition, the conductance limits on each of the cubic cell trap plates were used to effect electron beam alignment. Standard analog electronics include a high power excitation amplifier capable of $110-\mathrm{V}_{\mathrm{p}-\mathrm{p}}$ output, preamplifier, and cell controller.

All FTICR spectra were acquired from volatile samples introduced through high precision leak valves (Varian model 951-5106, Lexington, MA) and ionized by $70-\mathrm{eV}$ electron ionization. Specific trapping, excitation, and detection conditions are found in the figure captions for each type of experiment. The transfer of ions between cubic and single-trap electrode cells was achieved through appropriate gating of the three trap electrodes.

SIMION [62] plots were generated by a High Definition Systems (Sunnyvale, CA) 486DX/33 personal computer running DOS 5.0.

\section{Results and Discussion}

\section{Cell Theory and Performance}

The single-trap electrode cell depicted in Figure 1 illustrates the single annular trap electrode suspended in the center of the excitation and detection region. During consideration of the appropriate dimensions for the cell, the chief concern was the extent of the trap electrode along the $z$-axis because this dimension has a profound influence on system performance. As shown in Figure 2a, the flexibility of the single-trap electrode geometry permits a range of potential well depths along the cell centerline generated by varying the axial dimension of the trap electrode. The increasing well depth achieved as the trap electrode axial extent increases is analogous to that achieved by elongation of the cell in conventional two-trap electrode cells. As the potential well depth increases, the trapping well profile becomes increasingly particle-in-a-box and anharmonic. In addition, the excitation field decreases in homogeneity and magnitude at the center of the cell. Ions at the center of the cell $(z=0)$ are accelerated to smaller cyclotron radii due to the shielding of the excitation field by the trap electrode, with the excitation radius being dependent on preexcitation axial position. Reciprocity requires that the detection elec-

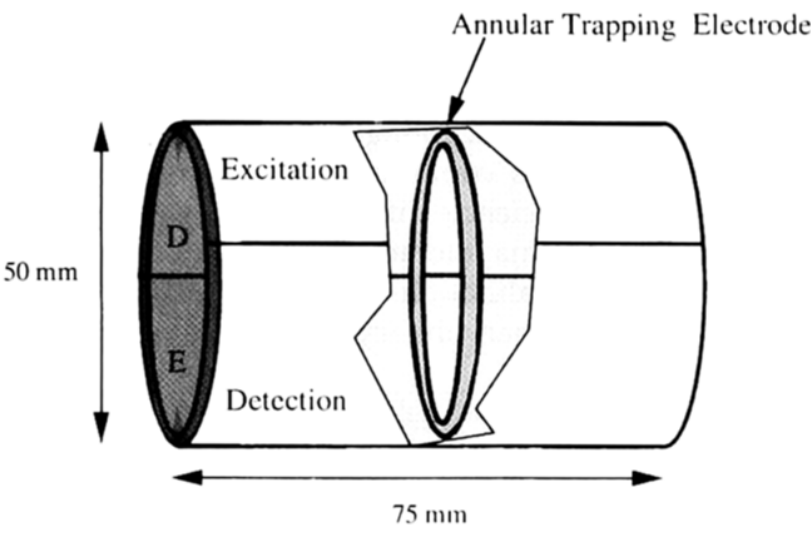

Figure 1. Diagram of the single-trap electrode cell. The annular trap electrode is positioned in the center of the excitation and detection region and a bias is applied opposite the ion charge to be trapped to form a potential well. The two sets of excitation and detection electrodes are employed as in the conventional FTICR cell.

trodes also must become increasingly shielded from the ion image current, which reduces sensitivity as more image charge strikes the trap electrode. Therefore, a compromise must be made between well depth and sensitivity due to shielding by the trap electrode. Our initial assessment was that a trap electrode of reduced axial extent would be superior even with a shallow trapping well. A radial electric field of reduced magnitude coincident with a trap electrode of reduced axial extent is essential for highest FTICR performance, and the loss in sensitivity and excitation field homogeneity from a trap electrode of increased axial extent would seriously limit performance.

A SIMION comparison of the single-trap electrode cell with the closed cubic cell and an aspect ratio 2 open elongated cell indicates that an applied trapping potential of $1.0 \mathrm{~V}$ yields a well depth of $0.67 \mathrm{~V}$ for the closed cubic cell and $0.80 \mathrm{~V}$ for the open elongated cell, whereas an applied trapping potential of $-1.0 \mathrm{~V}$ exhibits a well depth of $0.18 \mathrm{~V}$ for the single-trap electrode cell. Thus, the well depth for the single-trap electrode cell is 73 and $78 \%$ shallower at the cell centerline, respectively. Initially, there was concern that such a well depth would be too shallow to trap a sufficient number of ions to readily yield high sensitivity spectra. However, there was little reduction in the overall sensitivity between the single-trap electrode cell and the accompanying cubic cell. We attribute this to several unique features of the single-trap electrode geometry that includes the absence of axial ejection during excitation that negates the need for a deeper potential well when trapping low mass ions. In addition, as cyclotron radius increases, the potential well depth increases, which further reduces the likelihood of axial ejection.

The effect of the trap electrode on excitation field homogeneity using SIMION plots is shown in Figure $2 \mathrm{~b}$. An overlay of the centerline trapping well for a 3-mm trap electrode and the isopotential contour lines 


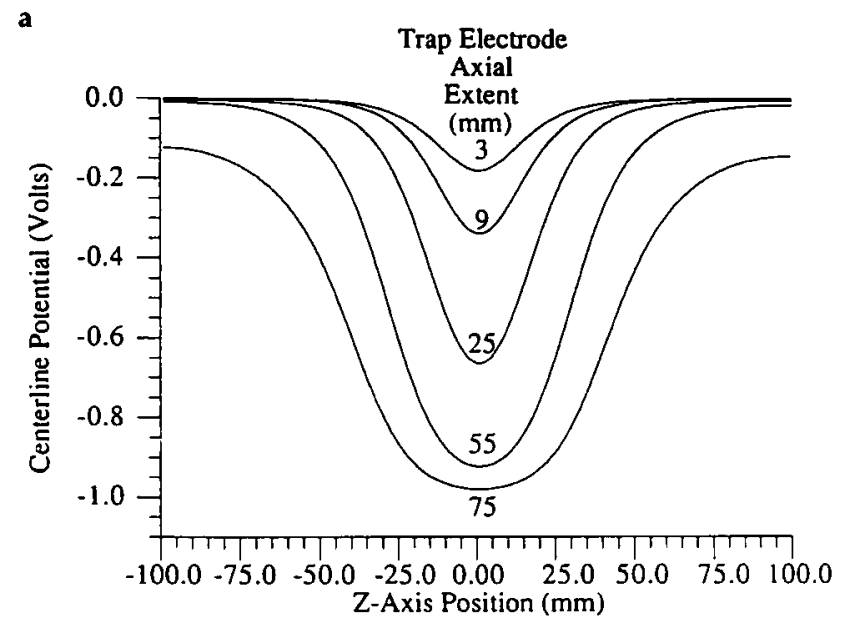

b

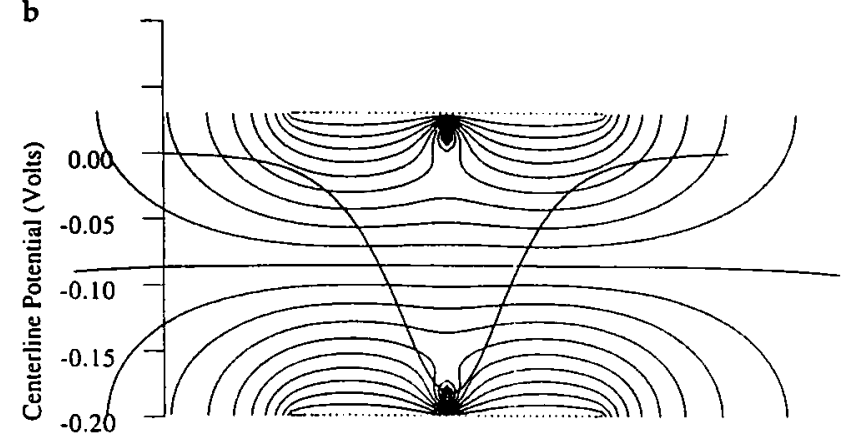

Figure 2. (a) SIMION plots that show the effect of increasing trap electrode extent along the z-axis on potential depression depth in volts. The $3-\mathrm{mm}$ trace represents the potential depression for the cell actually evaluated experimentally. (b) SIMION plots that depict the trapping potential along the cell centerline for a 3-mm trap electrode superimposed over the excitation isopotential lines formed when voltages $+V$ and $-V$ are applied to the excitation electrodes with the trapping and detection electrodes at ground potential. Ions are trapped in a potential well that remains in the homogeneous region of the excitation field.

generated when a voltage $+V$ is applied to one excitation electrode and $-V$ to the other excitation electrode while keeping the detection and trap electrodes at ground potential indicates that substantial excitation field curvature occurs in the vicinity of the trap electrode. However, along the cell centerline where ions are located prior to excitation, the excitation field is reasonably homogeneous and there is a negligible axial component to the excitation field. Interestingly, ions in the center of the trapping field that are accelerated to large radii do experience an increase in $z$-amplitude due to the axial component of the excitation field, but are translated into regions of the cell in which the axial component is substantially reduced. Additionally (Figure 3), a plot of potential versus $z$-axis position for various cell radii illustrates that the trapping potential well depth increases with cell radius due to increasing proximity to the trap electrode. Ions accelerated to large radii will be trapped in a deeper potential well compared to the center of the cell. At the cell centerline the well depth is only $18 \%$ of the applied potential, but

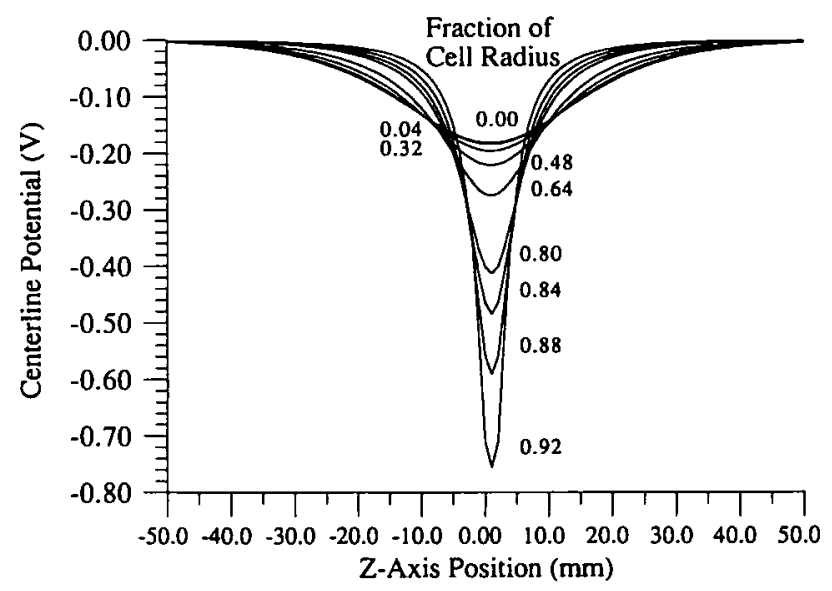

Figure 3. SIMION plots of potential versus axial position for various fractions of cell radius indicated by the numbers adjacent to the plot. The well depth increases exponentially with radius, and ions accelerated to large radius are less likely to be axially ejected due to the increased potential well depth in the vicinity of the trap electrode.

an ion at $92 \%$ of the cell radius encounters a potential well depth that is $75 \%$ of the applied potential, with the potential increasing exponentially as a function of radius. Experimentally, this should result in a decrease in axial ejection as ion radius increases.

An obvious concern with the single-trap electrode cell geometry is the effect on ions that travel in close proximity to the trap electrode during detection. In particular, it would be expected that ions at large cyclotron radii would encounter greater d.c. electric field inhomogeneity that would result in deterioration of FTICR performance manifested as signal reduction and peak broadening due to dephasing of the ion packet. The location of the annular trap electrode also could cause a substantial position dependent variation in the electric trapping field, which would promote errors in mass measurement or reduce resolving power. In addition, destabilization of the magnetron radius due to collisions or resistive cooling might become a more significant problem in the intensified radial electric field near the trap electrode, which would increase radial ion loss. However, experimentally it has been found that ions accelerated to modest cyclotron radii ( $<60 \%$ of the cell radius) can be detected at high mass resolving power. In addition, ions at small radii encounter only a modest radial electric field, and the growth in magnetron radius is expected to be minimal.

Presented in Figure 4a are SIMION radial electric field profiles for ions at the center of the trapping well as they are accelerated to increasing radii in the closed cubic, closed elongated, and the single-trap electrode cells. The cubic cell exhibits a linear increase in radial electric field to about $60 \%$ of the cell radius, whereas the closed elongated cell exhibits an almost negligible change in radial electric field throughout the entire cell radius. This is readily attributable to distance between the center of the potential well and the trap electrodes. 

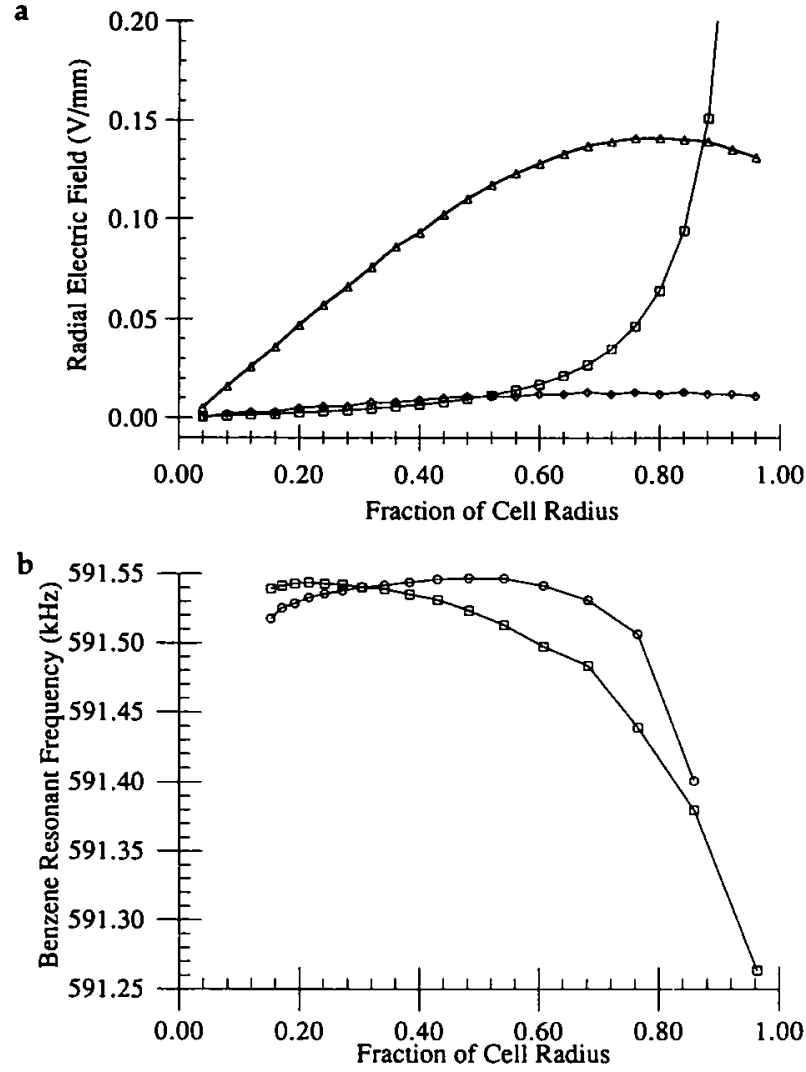

Figure 4. (a) Comparison of radial electric field magnitudes in cubic $(\Delta)$, elongated $(\diamond)$, and single-trap electrode cells $(\square)$ at increasing radius. Values are calculated for the center of the potential well $(z=0)$. (b) Experimentally determined cyclotron frequency for collisionally cooled benzene molecular ions accelerated to increasing radii by using single frequency resonance excitation for cubic $(O)$ and single-trap electrode cells $(\square)$.

Similarly, the single-trap electrode cell mimics the radial electric field of the closed elongated cell for more than $60 \%$ of the radial dimension because ions are relatively distant from the narrow annular trap electrode, which at this distance approximates a point change. However, beyond a radius of about $60 \%$, the radial electric field grows exponentially and cyclotron frequency depends increasingly on radial ion position, and the trap electrode approximates a line charge.

The radial electric field profile in Figure 4a suggests that as long as the ion radius is kept below $60 \%$ of the cell radius, FTICR performance should not deteriorate, and this is verified with three types of data. First, it was observed that unlike conventional cells, it was impossible to promote destabilization of the magnetron orbit. Trapping potential, ion number, and neutral pressure values that increase the magnetron radius and lead to rapid radial ejection in the cubic cell have no apparent effect on magnetron radius in the singletrap electrode cell. This can be attributed to the negligible radial electric field experienced by ions formed along the centerline of the cell. Experimental data shown in Figure $4 \mathrm{~b}$ to determine the frequency shift for the single-trap electrode cell as a function of trap- ping potential also indicates that for ions with radii below $40 \%$ of the cell dimension, there is effectively no change in cyclotron frequency. Comparison data with the cubic cell indicate the superiority of the single-trap electrode cell at very small radius where the cyclotron radius is constant, although with the cubic cell, less cyclotron frequency variation is measured over a larger region of the cell radius. Both cells exhibit a significant reduction in detected cyclotron frequency for ions with large radii that are in proximity to the cell perimeter.

A comparison of relative perfluorotributylamine (PFTBA) fragment ion abundances is often used to evaluate the extent of axial ejection, because low mass ion abundance is reduced at larger excitation voltages. Excitation in the single-trap electrode cell was compared experimentally to the cubic cell and evaluated for axial ejection. Presented in Figure 5 are representative excitation profiles of PFTBA collected in the cubic (Figure $5 a$ ) and the single-trap electrode (Figure $5 b$ ) cells at the applied trapping potential of $+0.50 \mathrm{~V}$ (effective well depth of $0.38 \mathrm{~V}$ ) and $-0.50 \mathrm{~V}$ (effective
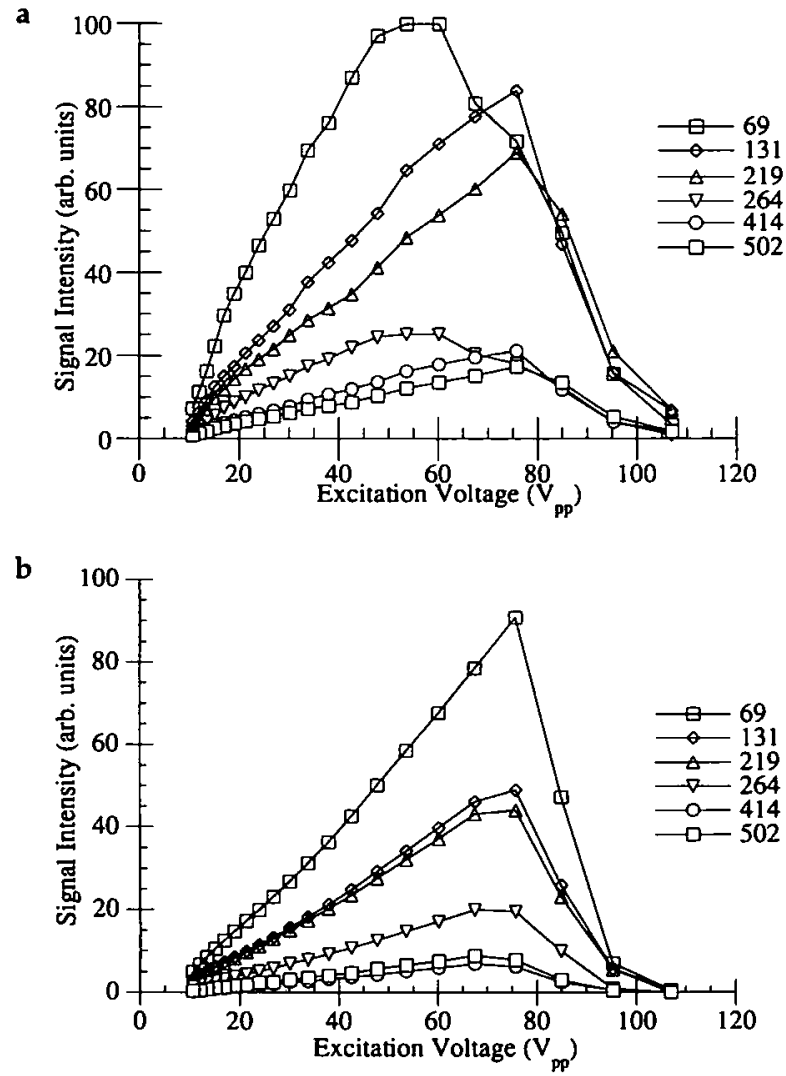

Figure 5. (a) Excitation profiles for PFTBA dissociation fragments formed by electron ionization at an energy of $-70 \mathrm{eV}$ in a cubic cell with $+0.5-\mathrm{V}$ applied trapping potential (well depth of $0.33 \mathrm{~V}$ ) that indicates ejection of the $m / z 69$ and 131 fragment ions at large excitation energies. (b) PFTBA fragments in the single-trap electrode cell with $-0.5-\mathrm{V}$ applied trapping potential (well depth of $0.09 \mathrm{~V}$ ). The mass independent linear increase in signal magnitude with increasing cyclotron radius verifies that axial ejection does not occur even at an applied excitation voltage that corresponds to radial ejection at the cell perimeter. 
well depth of $0.09 \mathrm{~V}$ ), respectively. The data for the single-trap electrode cell indicate that axial ejection is virtually nonexistent, because ions are confined electrostatically to a region where the excitation field does not impart a significant axial component to the ion trajectory. Similar experimental conditions in the conventional cubic cell yield spectra in which low mass ions $(m / z 69,131)$ are absent at the larger excitation voltages, indicative of axial ejection in which ions encounter axial components of the excitation field at large $z$-displacements [63-66]. In addition, the position of the trap electrode does not appear to affect the mass independent linearity of signal intensity with excitation voltage.

Historically, axial ejection has been reduced or eliminated successfully by increasing the homogeneity of the excitation field by distribution of the excitation field through a voltage divider circuit to a grid in closed cells [16-18,67] or by capacitively coupling the excitation and trap electrodes in open cell designs [42]. However, each of these techniques requires a substantial increase in the complexity of the cell. The singletrap electrode cell is exceptional in that the number of electrodes is one less than the conventional cell design, yet the cell design minimizes the long-standing problem of axial ejection associated with FTICR.

Data observed in conventional two-trap electrode cells support the hypothesis that high mass resolving power is better achieved at smaller excitation radii where less electric field inhomogeneity is encountered. This also is exhibited in the single-trap electrode cell. A heterodyne spectrum of the benzene molecular ion was obtained after formation in the source cubic cell by electron ionization and transfer to the single-trap electrode cell. Ions were allowed to axially cool for $10 \mathrm{~s}$ before excitation. The apodized magnitude mode resolution was $1.45 \times 10^{6}$ obtained at $40 \%$ of the cell radius at a pressure of $3 \times 10^{-9}$ torr with $64 \mathrm{~K}$ data points acquired over a bandwidth of $3.5 \mathrm{kHz}$ with a 6-s transient lifetime. This represents the highest resolving power achieved in this magnet and vacuum chamber and provides, at least in a qualitative manner, an indication that the geometric arrangement of the trap electrode at the center of the cell is not a detriment to high performance FTICR. The data also indicate that with appropriate experimental prudence, high resolution FTICR can be performed in cells that have electrode geometries that result in a greater deviation from an optimal quadrupolar potential.

Sensitivity is an important consideration for the design and construction of new cell geometries. Elongated cells were designed chiefly to reduce the radial electric field and increase ion capacity, but it was later discovered that greater sensitivity could be achieved in elongated cells in which less ion image current terminates on the trap electrodes [68]. Because ions cooled to the center of the cell are more distant from the trap electrodes, more image current is collected on the detection electrodes. Similarly, for the single-trap elec- trode geometry, the small axial extent of the trap electrode limits the amount of image current that is lost on the trap electrode.

SIMION was used in Figure 6 to ascertain the relative amount of image current collected on the detection electrodes in the cubic and single-trap electrode cells as a function of ion radius and axial position. Because the detection geometry is the same as the excitation geometry, only rotated by $90^{\circ}$, the fraction of image charge induced by a point charge in the detection plane can be obtained by evaluation of the excitation potential in the excitation plane (cf. Figure 2b). Ions located at maximum radius in the cubic cell induce greater signal on the detection electrodes compared to the single-trap electrode cell. This is expected due to the shielding of the detection electrodes by the trap electrode in the single-trap electrode cell. Overall, the single-trap electrode cell exhibits lower sensitivity than the cubic cell when ions are accelerated to maximum radius because more current is lost on the annular trap electrode. However, at intermediate and lower ion radii the signal induced on the detection electrodes is greater in the single-trap electrode cell than in the cubic cell. This is attributed to greater current lost on the trap electrodes in the cubic cell, whereas current lost on the trap electrode in the single-trap electrode cell is reduced as the ion radius decreases. At ion positions of increased axial displacement near the trap electrodes in the cubic cell, the detected signal intensity is attenuated due to image charge striking the trap electrodes. However, at increased ion axial displacement in the single-trap electrode cell, image current is

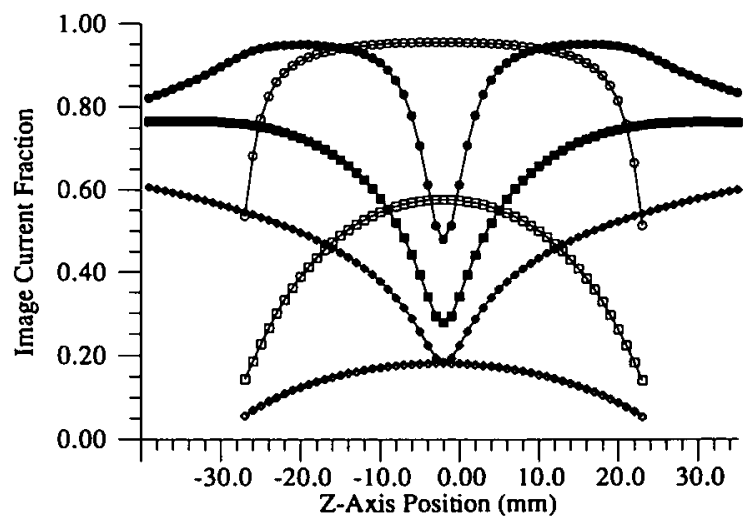

Figure 6. SIMION plot that predicts the relative amount of image current collected on the detection electrodes for a cubic cell at maximum $(O)$, intermediate $(\square)$, and minimum $(\diamond)$ radius and for a single-trap electrode cell at maximum (O), intermediate $(\boldsymbol{\nabla})$, and minimum $(\downarrow)$ radius as a function of axial position. Ions at maximum radius in the cubic cell induce greater signal on the detection electrodes compared to the single-trap electrode cell due to the shielding of the detection electrodes by the trap electrode. At reduced radius more image current is measured on the single-trap electrode cell detection electrodes compared to the cubic cell because more signal strikes the trap electrodes in the cubic cell. The reduction in detected signal at increased axial dispersion is more severe in the cubic cell than in the single-trap electrode cell. 
not attenuated due to the lack of trap electrodes at the axial perimeters of the cell.

\section{Trapping Externally Generated Ions in the Single- Trap Electrode Cell}

The mechanism for trapping externally generated ions in the single-trap electrode cell differs fundamentally from conventional dual-trap electrode cells in that no potential barrier exists to impede ion introduction to the cell. As shown in Figure 7a, introduction of externally generated ions into a conventional two-trap electrode cell that has a potential well formed by two electrodes at higher potential than the center of the trapping region depends on the possession of sufficient translational energy to overcome the repulsion of the trap electrode biased at the same polarity as the ion. However, in the single-trap electrode cell in Figure $7 b$, where the center of the trapping region is at negative potential, all ions regardless of their translational energies penetrate the trapping region and, consequently, have the opportunity to be trapped. This suggests that, in principle, the primary source of kinetic energy discrimination for external ion injection in the conventional cell is avoided in a single-trap electrode cell without the need to employ gated trapping techniques where the trap voltage is momentarily held at ground

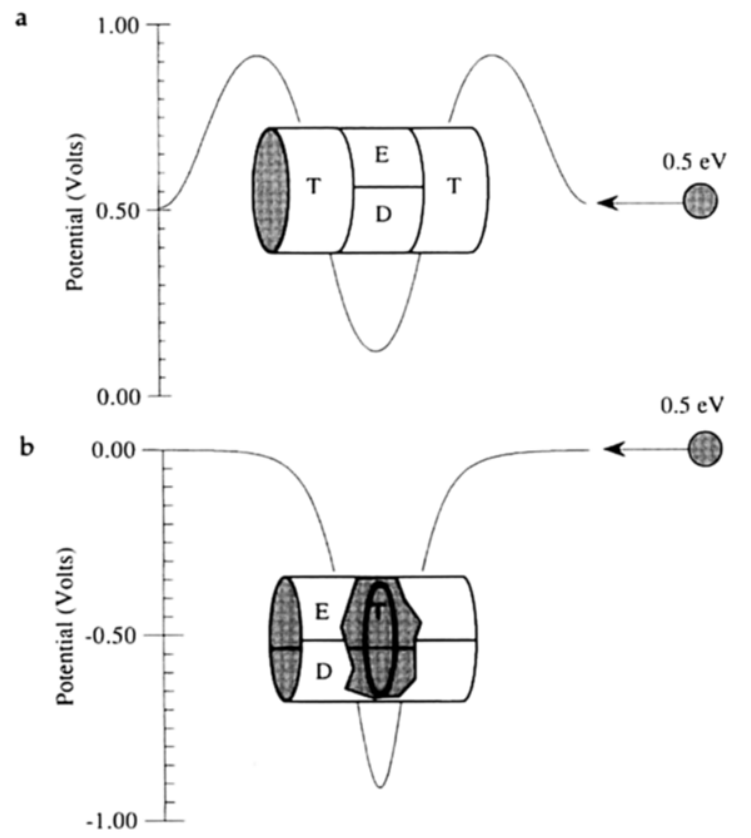

Figure 7. Comparison of ion trapping for externally formed $0.5-\mathrm{eV}$ ions in (a) a conventional two-trap electrode cell and (b) the single-trap electrode cell. In the conventional two-trap electrode cell, an ion is repulsed by the potential barrier that exists unless the trap voltage is lowered or gated trapping is performed. In the single-trap electrode cell, all ions penetrate the cell regardless of the ion energy or the trap potential. Whether an externally formed ion is trapped in the single-trap electrode cell depends upon its final translational energy after interaction with various energy loss mechanisms. to allow ions to penetrate the cell, after which it is raised.

The limitation of gated trapping in conventional cells is the inability to efficiently accumulate ions if the ionization duty cycle is low compared to the trapping oscillation period. In these cases, ions are quickly lost from the cell if full trapping potentials are not reimposed within a few hundred microseconds following injection. In addition, ion loss may occur if the trapping potentials are reinstated nonadiabatically, because additional energy imparted during the rapid increase in trap potential causes ion loss if the total ion energy becomes greater than the well depth [69]. However, the single-trap electrode cell trapping mechanism exhibits simultaneous application of gated and accumulated trapping because ions of very low translational energy can continuously penetrate the trapping volume regardless of the ionization duty cycle.

A distinction can be drawn in the selection of parameters for external ion injection between the two cell designs. If accumulated trapping is to be used to trap injected ions in a conventional cell, the trapping potential must be matched to the average kinetic energy of the injected ion cloud. Ions that enter the cell and have excess kinetic energy subsequently experience sufficient collisional stabilization or redistribution of their translational motion into transverse motion due to electric or magnetic field inhomogeneities from cell electrodes so that they can be retained. Collisional cooling with a pulsed background gas is often used to enhance intracell cooling. In contrast, all ions enter the single-trap electrode cell, regardless of the applied trapping potential or the kinetic energy of the injected ion population. Once inside the cell, ion trapping is again independent of the applied potential and depends solely on the kinetic energy of the ion that approaches the cell and the efficiency of the energy-loss mechanisms. As a consequence, this unique trapping scheme favors lower energy populations of injected ions.

The simplicity of the single-trap electrode cell is in the creation of a potential well without application of a negative d.c. potential to the excitation or detection electrodes while the trap electrodes are held at ground, which could be achieved in two-trap electrode cells to perform gated and accumulated trapping, but which would require a switch to a transfer trapping function to the trap electrodes during excitation and detection. The single-trap electrode cell also eliminates the need to apply a sinusoidal waveform centered at ground to the trap electrodes to trap low energy ions, which would present a trap admittance of only narrow energy aperture for short duration and thereby reduce the effective duty cycle of the trap.

It is possible to apply gated trapping to retain higher energy ions in the single-trap electrode cell without reliance on extensive collisional cooling. Application of a decelerating voltage to the trap electrode that is of the same polarity as the ions to be trapped 
results in the potential hill that will reduce the ion translational velocity. The polarity of the trap electrode can then be switched after a suitable time to trap ions in the well that is formed. Equivalently, the trap electrode can be left at ground until after the ion cloud has passed, whereupon it can be pulsed to an appropriate voltage of opposite polarity to the ion [70] to affect ion deceleration and trapping.

The availability of a conventional cubic cell positioned in the source region provided an opportunity to compare performance characteristics of the two cells as well as observe the controlled transfer of ions into the single-trap electrode cell. In a first set of experiments designed to mimic an external ion source, ions formed in the cubic cell by electron ionization were transferred to the single-trap electrode cell. As shown in Figure 8a, the applied potential to affect external injection was controlled to deliver ion populations of well defined kinetic energy to the cell. Ions were first cooled for 100 $\mathrm{ms}$ to reach thermal equilibrium in the source cubic cell, followed by grounding the conductance limit so the source trap electrode behaved as an acceleration grid. In these experiments, the single-trap electrode

a

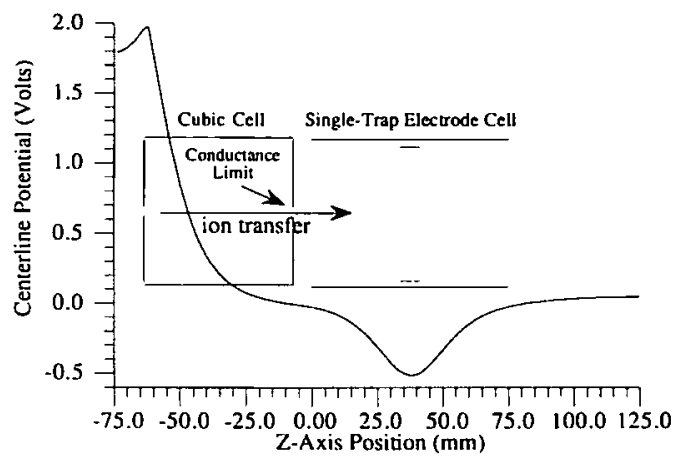

b

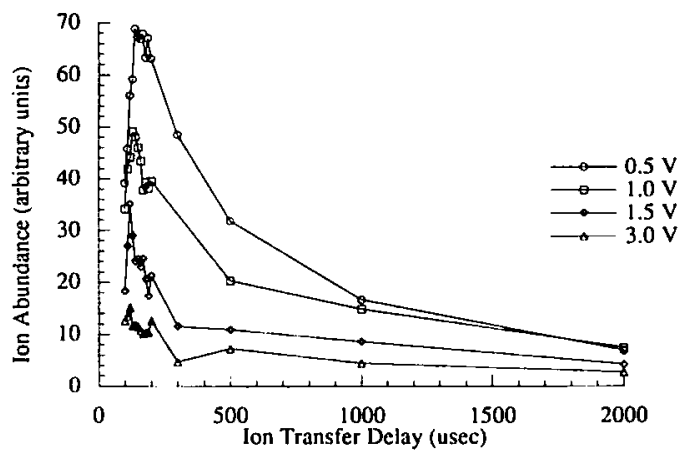

Figure 8. (a) SIMION representation of potentials used for transfer of ions from the cubic source cell to the analyzer singletrap electrode cell. Ions formed initially in the cubic cell by electron ionization are transferred to the single-trap electrode cell by grounding the conductance limit and operating the source trap plate as an acceleration grid. In these experiments, the potential on the trap electrode in the single-trap electrode cell is switched after an appropriate ion transfer time from ground potential to the desired trapping potential. (b) Plot of benzene ion abundance versus ion transfer time for $0.5-\mathrm{V}(\mathrm{O}), 1.0-\mathrm{V}(\square)$, $1.5-\mathrm{V}(\diamond)$, and $3.0-\mathrm{V}(\Delta)$ transfer voltages. Ion trapping improves substantially for ions injected with lower kinetic energy. cell was initially at ground potential and then gated to $-2 \mathrm{~V}$ to determine optimum transfer time as a function of ion abundance. Presented in Figure $8 \mathrm{~b}$ are ion abundance profiles as a function of transfer time for several acceleration voltages. In all cases, essentially the same number of ions enter the cell. As would be expected, the cell discriminates in favor of ions with the lowest initial kinetic energy because these ions are preferentially trapped by collisional cooling.

A final experiment was performed to gain a better understanding of the extent to which radial dispersion of the ion cloud occurs due to magnetron effects. As shown in Figure 9a, electrode potentials were applied to the source and analyzer cells to create a potential depression between the two vacuum regions, which permitted the shuttling of ions between cells. In this case, a positive potential was applied to the single-trap

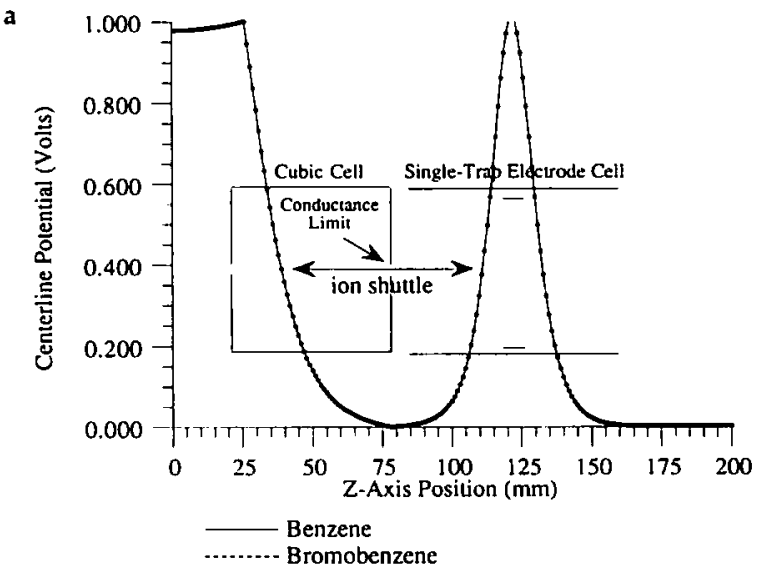

b

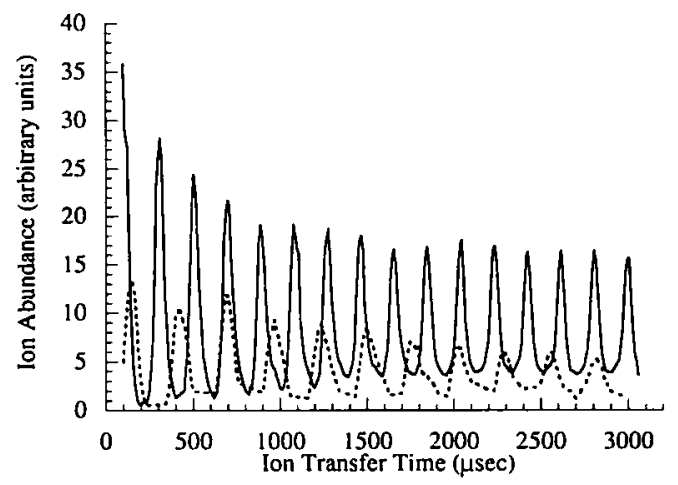

Figure 9. (a) SIMION representation of potentials used to shuttle ions between the source cubic cell and analyzer single-trap electrode cell. The source trap of the cubic cell was maintained at $+2.0 \mathrm{~V}$, the conductance limit was gated to ground, and a positive potential of $+3.0 \mathrm{~V}$ was applied to the single-trap electrode cell to form an extended well to permit shuttling of positive ions between cells. After transfer, the single-trap electrode cell was switched to an appropriate negative trapping potential for ion cietection. (b) Profiles of ion magnitude as a function of transfer time for benzene ( - ) and bromobenzene (-. - - ) molecular ions formed by electron ionization in the source cubic cell and detected in the analyzer single-trap electrode cell. The calculated trapping periods were $192 \mu$ s for benzene and 265 $\mu \mathrm{s}$ for bromobenzene molecular ions, which are in close agreement with the expected square root of mass dependence. 
electrode cell to redirect positive ions back to the source region. The shuttling of benzene and bromobenzene molecular ions is demonstrated in Figure $9 \mathrm{~b}$. The transfer periods for benzene $(\mathrm{m} / \mathrm{z} 78)$ and bromobenzene $(m / z 156)$ was 192 and $265 \mu \mathrm{s}$, respectively, which are in close agreement with the expected square root of mass dependence. In the data shown, sufficient time ( $3 \mathrm{~ms}$ ) was allowed for benzene ions to shuttle between the two cells 16 times. The initial reduction in ion abundance is because of radial dispersion of ions formed in the source cell due to space-charge and clipping of the conductance limit that separates the two cells. After this initial reduction, ion transfers between cells were achieved with minimal signal loss, which indicates that the effect of the radial electric field on the magnetron radius is insignificant over the time scale of the experiment.

Although the performance of the single-trap electrode cell is notable in relation to axial ejection and radial electric field, an immediate improvement to the cell design will be attempted that should overcome several limitations of the prototype cell related to the nonlinear excitation field. With the excitation electrodes capacitively coupled to the segmented trap electrode, improved excitation field uniformity will occur, which should result in the independence of the ion radius from the preexcitation $z$-axis position. In addition, capacitive coupling should improve excitation efficiency because more energy will be available to the ions with the trap electrode no longer shielding the excitation field.

\section{Conclusions}

The flexibility of the open cell geometry allows the construction of a single-trap electrode cell that achieves many of the desirable attributes traditionally attained in more complex cell designs. For example, the imposition of the trapping well within the homogeneous excitation region permits ions to be accelerated to large radius without axial ejection. The reduction in magnetron effects achieved in elongated cells also is realized in the single-trap electrode cell with a trap electrode of reduced axial extent because the radial electric field is negligible to $60 \%$ of the cell radius. As a further indication of the adequate performance of the trapping field, the cell is capable of high mass resolving power. Finally, the single-trap electrode trapping well permits all externally formed ions-regardless of kinetic energy - to penetrate the cell, as in gated trapping, yet allows continuous ion injection that is characteristic of accumulated trapping.

Future work will concentrate on exploitation of the unusual trapping characteristics of the single-trap electrode cell for externally generated ions by evaluation of several properties of the applied excitation and trapping fields. In addition, an improvement in the uniformity of the excitation field will be achieved with capacitive coupling.

\section{Acknowledgments}

This work was supported by the Welch Foundation (F-1138), the National Institutes of Health, the Texas Advanced Research Program, and the National Science Foundation (CHE9013384 and CHE9057097). In addition, V. H. V. wishes to thank J. Scott Anderson for many helpful discussions as well as assistance with the manuscript.

\section{References}

1. Comisarow, M. B.; Marshall, A. G. Chem. Phys. Lett. 1974, 25, 282-283.

2. Comisarow, M. B. J. Chem. Phys. 1978, 69, 4097-4104.

3. Comisarow, M. B. Int. I. Mass Spectrom. Ion Phys. 1981, 37, 251-257.

4. McIver, R. T., Jr. Rev. Sci. Instrum. 1970, 41, 555-558.

5. Hunter, R. L.; Sherman, M. G.; McIver, R. T., Jr. Int. J. Mass Spectrom. Ion. Processes 1983, 50, 259-273.

6. Lee, S. H.; Wanczek, K.-P.; Hartmann, H. Adv. Mass Spectrom. 1980, 88, 1645-1649.

7. Kofel, P.; Alleman, $\mathrm{M}_{\text {; }}$ Kellerhans, H. P.; Wanczek, K.-P. Int. J. Mass Spectrom. Ion Processes 1986, 74, 1-12.

8. Gabrielse, G.; Mackintosh, F. C. Int. J. Mass Spectrom. Ion Processes 1984, 57, 1-17.

9. Schweikhard, L.; Lindinger, M.; Kluge, H.-J. Rev. Sci. Instrum. 1990, 61, 1055-1058.

10. Rempel, D. L.; Ledford, E. B., Jr.; Huang, S.; Gross, M. L. Anal. Chem. 1987, 59, 2527-2532.

11. Van Dyck, R. S., Jr.; Schwinberg, P. B. Phys. Rev. Lett. 1980, 47, 395-398.

12. Brown, L. S.; Gabrielse, G. Rev. Mod. Phys. 1986, 58, 233-311.

13. Schweikhard, L.; Lindinger, M.; Kluge, H.-J. Int. J. Mass Spectrom. Ion Processes 1990, 98, 25-33.

14. Ledford, E. B., Jr.; Ghaderi, S.; White, R. L.; Spencer, R. B.; Kulkarni, P. S.; Wilkins, C. L.; Gross, M. L. Anal. Chem. 1980, $52,463-468$.

15. Grosshans, P. B.; Wang, M.; Marshall, A. G. Proc. 36th ASMS Conference on Mass Spectrometry and Allied Topics; San Francisco, CA, 1988; pp 592-593.

16. Wang. M.; Marshall, A. G. Anal. Chem. 1990, 62, 515-520.

17. Hanson, C. D.; Castro, M. E.; Kerley, E. L.; Russell, D. H. Anal. Chem. 1990, 62, 520-526.

18. Caravatti, P.; Allemann, M. Org. Mass Spectrom. 1991, 26, 514-518.

19. Grosshans, P. B.; Marshall, A. G. Proc. 39th ASMS Conference on Mass Spectrometry and Allied Topics; Nashville, TN, 1991; Pp 49-50.

20. Chen. R.; Grosshans, P. B.; Limbach, P. A.; Marshall, A. G. Proc. 40th ASMS Conference on Mass Spectrometry and Allied Topics; Washington, DC, 1992; pp 1733-1734.

21. Wang, M.; Marshall, A, G. Anal. Chem. 1989, 61, 1288-1293.

22. Naito, Y.; Fujiwara, M.; Inoue, M. Int. J. Mass Spectrom. Ion Processes 1992, 120, 179-192.

23. Bollen, G.; Hartmann, H.; Kluge, H.-J.; König, M.; Otto, T.; Savard, G.; Stolzenberg, H. Phys. Scripta 1992, 46 581-586.

24. Rempel, D. L.; Grese, R. P.; Gross, M. L. Int. J. Mass Spectront. Ion Processes 1990, 100, 381-395.

25. Jacoby, C. B.; Holliman, C. L.; Rempel, D. L.; Gross, M. L. J. Am. Soc. Mass Spectrom. 1993, 4, 186-189.

26. Jeffries, J. B.; Barlow, S. E.; Dunn, G. H. Int. J. Mass Spectrom. Ion Processes 1983, 54, 169-187.

27. Mitchell, D. W. Int. J. Mass Spectrom. Ion Processes 1991, 107, 417-434.

28. Kerley, E. L.; Hanson, C. D.; Castro, M. E.; Russell, D. H. Anal. Chem. 1989, 61, 2528-2534. 
29. Byrne, J.; Farago, P. S. Proc. Phys. Soc. London 1965, 86, 801-815.

30. Malmberg, J. H.; deGrassie, J. S. Phys. Rev. Lett. 1975, 35 , 577-580.

31. deGrassie, J. S.; Malmberg, J. H. Phys. Rev. Lett. 1977, 39, 1077-1080.

32. deGrassie, J. S.; Malmberg, J. H. Phys. Fluids 1980, 23, 63-81.

33. Gabrielse, G.; Rolston, S. L.; Haarsma, L. Phys. Lett. A 1988, $129,38-42$.

34. Gabrielse, G.; Haarsma, L.; Rolston, S. L. Int. J. Mass Spectrom. Ion Processes 1989, 88, 319-332.

35. Quint, W.; Kaiser, R.; Hall, D.; Gabrielse, G. Hyperfine Interact. 1993, 76, 181-188.

36. Beu, S. C.; Laude, D. A., Jr. Int. I. Mass Spectrom. Ion Processes 1992, 112, 215-230.

37. Schweikhard, L.; Lindinger, M.; Hartmann, H. Proc. $41 s t$ ASMS Conference on Mass Spectroscopy and Allied Topics; San Francisco, CA, 1993; pp 694a-b.

38. Dearden, D. V.; Chu, I. H.; Zhang, $\mathrm{H}_{\text {; }}$ Chen, Q. Proc. 40th ASMS Conference on Mass Spectrometry and Allied Topics; Washington DC, 1992; pp 71-72.

39. Marto, J. A.; Guan, S.; Marshall, A. G. Rapid Commun. Mass Spectrom. 1994, 8, 615-620.

40. Beu. S. C.; Senko, M. W.; Quinn, J. P.; Wampler, F. M., III; McLafferty, F. W. J. Am. Soc. Mass Spectrom. 1992, 4, 557-565.

41. Huang, Y.; Kim, H. S.; Jackson, G.; Guan, S.; Marshall, A. G. Proc. 42nd ASMS Conference on Mass Spectrometry and Allied Topics; Chicago, IL, 1994; $\mathrm{p} 723$.

42. Beu, S. C.; Laude, D. A., Jr. Anal. Chem. 1992, 64, 177-180.

43. Vartanian, V. H.; Laude, D. A. Org. Mass Spectrom. 1994, 29, 692-694.

44. Vartanian, V. H.; Laude, D. A. Proc. 42nd ASMS Conference on Mass Spectrometry and Allied Topics; Chicago, IL, 1994; 726.

45. Kingdon, K. H. Phys. Rev. 1923, 21, 408-418.

46. Douglas, R. A.; Zabritski, J.; Herb, R. G. Rev. Sci. Instrum. $1965,36,1-6$.

47. Hooverman, R. H. J. Appl. Phys. 1963, 34, 3505-3508.

48. Limbach, P. A.; Marshall, A. G. Int. I. Mass Spectrom. Ion Processes 1993, 125, 135-143.

49. Solouki, T.; Gillig, K. J.; Russell, D. H. Anal. Chem. 1994, 66, 1583-1587.

50. Bolanos, B. J.; Schmidt, E. G.; Anderson, J. S.; Herath, P.; Gupta, R. V.; Hendrickson, C. J.; Laude, D. A. Proc. 42nd ASMS Conference on Mass Spectrometry and Allied Topics; Chicago, IL, 1994; p 745.
51. Baker, F. A.; Hasted, J. B. Philos. Trans. Roy. Soc. 1966, 261 33-65.

52. Hendrickson, C. L.; Hadjarab, F.; Laude, D. A., Jr. Int. J Mass Spectrom. Ion Processes 1995, 141, 161-170.

53. Kofel, P.; Allemann, M.; Kellerhals, H. P.; Wanczek, K. P. Int. J. Mass Spectrom. Ion Processes 1986, 72, 15-31.

54. Beu, S. C.; Laude, D. A., Jr. Int. J. Mass Spectrom. Ion Processes 1991, 104, 109-127.

55. Castoro, J. A.; Koster, C.; Wilkins, C. Rapid Commun. Mass Spectrom. 1992, 6, 239-241.

56. Hunt, D. F.; Shabanowitz, J.; Mclver, R. T., Jr.; Hunter, R. L.; Syka, J. E. P. Anal. Chem. 1985, 57, 765-768.

57. Alford, J. M.; Williams, P. E.; Trevor, D. J.; Smalley, R. E. Int I. Mass Spectrom. Ion Processes 1986, 72, 33-51.

58. Weddle, G. H.; Dunbar, R. C. I. Phys. Chem. 1988, 92, 5706-5709.

59. Moore, R. B.; Lunney, M. D. N.; Rouleau, G.; Savard, G.; The ISOLDE Collaboration. Physica Scripta 1992, 46, 569-574.

60. Beu, S. C.; Laude, D. A., Jr. Int. I. Mass Spectrom. Ion Processes 1990, 97, 295-310.

61. Bamberg, M.; Wanczek, K. P. Proc. 37th ASMS Conference on Mass Spectrometry and Allied Topics; Miami Beach, Fl, 1987; P 456.

62. Dahl, D. A.; Delmore, J. E. SIMION PC/PS2 Version 4.0, Report EGG-CS-7233, Rev. 2, Idaho National Engineering Laboratory, Idaho Falls, ID, April 1988.

63. Rempel, D. L.; Huang, S. K.; Gross, M. L. Int. J. Mnss Spectrom. Ion Processes 1986, 70, 163-184.

64. Huang, S. K.; Rempel, D. L.; Gross, M. L. Int. J. Mass Spectrom. Ion Processes 1986, 72, 15-31.

65. Van der Hart, W. J.; Van der Guchte, W. J. Int. I. Mass Spectrom. lon Processes 1988, 82, 17-31.

66. Van der Guchte, W. J.; Van der Hart, W. J. Int. J. Mass Spectrom. Ion Processes 1990, 90, 317-326.

67. Sommer, H.; Thomas, H. A.; Hipple, J. A. Phys. Rev. 1951, 82, 697-702.

68. Grosshans, P. B.; Shields, P. J.; Marshall, A. G. I. Chem. Phys. 1991, 94, 5341-5352.

69. Hofstadler, S. A.; Laude, D. A., Jr. Int. J. Mass Spectrom. Ion Processes 1990, 101, 65-78.

70. Maruyama, S.; Anderson, L. R.; Smalley, R. E. Rev. Sci. Instrum. 1990, 61, 3686-3693. 